\title{
El control de la presión arterial en la consulta de enfermedad renal crónica avanzada
}

\author{
Francisco Cirera Segura, Álvaro Pérez Baena, Nuria Sánchez Flores, Jesús Lucas Martín Espejo \\ Enfermero/a, Unidad de Gestión Clínica Uro-Nefrológica.H.U. Virgen del Rocío.Sevilla
}

\section{Resumen}

Nos propusimos valorar los resultados obtenidos, tras la formación e intervenciones de enfermería realizadas para mejorar la presión arterial, en pacientes con enfermedad renal crónica avanzada.

\section{Material y Método}

Se realizó un estudio descriptivo retrospectivo, de 6 meses de duración. Se estudiaron todos los pacientes incidentes en la consulta de enfermedad renal crónica avanzada.

Se recogieron los datos relacionados con la enfermedad renal crónica avanzada y su evolución, en la consulta inicial, a los tres y seis meses.

\section{Resultados}

La muestra la formaron un total de 148 pacientes, de los cuales el $60,8 \%$ eran hombres. La edad media fue $68,7 \pm 14,8$ años.

Evaluamos los cambios en las constantes vitales relacionadas con la presión arterial a lo largo del estudio, obteniendo diferencias significativas para la presión arterial sistólica $(p<0,001)$, la presión arterial diastólica $(p<0,001)$ y para la presión arterial media $(p<0,001)$, no siendo así para la frecuencia cardiaca $(p=0,307)$.

El número de hipotensores disminuyó a lo largo del estudio sin significación estadística. Existió una diferencia significativa en el peso de los pacientes pasando de $80,1 \pm 16,7 \mathrm{~kg}$. a $77,6 \pm 16,1 \mathrm{~kg}$. al final del seguimiento $(p<0,001)$. La presencia de edemas varió de un $26,1 \%$ a un $18,8 \%$ al concluir el estudio $(p=0,052)$.

Analizamos el sodio en orina $(p=0,665)$ y observamos que no hubo diferencias significativas. Sí encontramos cambios significativos en el hábito tabáquico $(p=0,004)$ pero no en el consumo de alcohol $(p=0,5)$.

Correspondencia:

Francisco Cirera Segura

C/ Ángel Ripoll Pastor, 4-6 $\mathrm{A}$

41006 Sevilla

E-mail: paco.cirera@gmail.com

\section{Conclusiones}

Las actividades de enfermería han conseguido mejorar el control de la presión arterial, a los pacientes incidentes en la consulta enfermedad renal crónica avanzada. Estas intervenciones deben ser una constante en el tratamiento del paciente hipertenso con enfermedad renal crónica avanzada.

\section{PALABRAS CLAVE:}

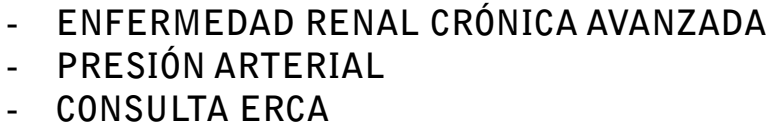

\section{Blood pressure monitoring in advanced chronic renal disease nursing controls}

\section{Objective}

We set out to evaluate the results obtained, after training and nursing interventions carried out to improve blood pressure, in patients with advanced chronic renal disease.

\section{Material and Method}

A 6-month long retrospective descriptive study was carried out. All patients in the advanced chronic renal disease clinic were studied.

Data relating to the advanced chronic renal disease and its evolution were gathered at the first visit, and then after three and six months.

\section{Results}

The sample was made up of a total of 148 patients, $60.8 \%$ of whom were men. The average age was 68.7 \pm 14.8 years.

We assessed the changes in vital constants related to blood pressure throughout the study, obtaining significant differences for systolic pressure $(p<0.001)$, diastolic pressure $(p<0.001)$ and for mean blood pressure $(p<0.001)$, but not for heart rate $(p=0.307)$.

The number of hypotensive medications fell throughout the study without statistical relevance. There was a significant difference in patients' weight from 
$80.1 \pm 16.7 \mathrm{~kg}$ to $77.6 \pm 16.1 \mathrm{~kg}$ at the end of the monitoring $(p<0.001)$. The presence of oedemas varied from $26.1 \%$ to $18.8 \%$ by the end of the study ( $p=0.052$ ).

We analysed sodium in urine $(p=0.665)$ and observed that there were no significant differences. We did however find significant changes in smoking habits $(p=0.004)$ but not in alcohol consumption $(p=0.5)$.

\section{Conclusions}

Nursing activities have managed to improve the control of blood pressure in patients attending the advanced chronic renal disease nursing control. These interventions should be a constant in the treatment of hypertensive patients with advanced chronic renal disease.

\section{KEY WORDS:}

\section{- ADVANCED CHRONIC RENAL DISEASE \\ - BLOOD PRESSURE \\ - ACRD CLINIC}

\section{Introducción}

Un control inadecuado de la presión arterial (PA) contribuye al deterioro de la función renal ${ }^{1-2}$, sobre todo en las fases finales de la enfermedad renal crónica avanzada (ERCA).

La información, la formación y las intervenciones dirigidas a los pacientes con hipertensión ${ }^{3-4}$, puede favorecer un mejor control de la misma así como beneficiar el enlentecimiento del deterioro renal y forman parte de las actividades de enfermería en la consulta ERCA.

Como objetivo de este trabajo, nos propusimos valorar los resultados obtenidos, tras la información, formación e intervenciones de enfermería realizadas para la mejora de la presión arterial, en los pacientes incidentes en la consulta ERCA.

\section{Material y métodos}

Se realizó un estudio descriptivo retrospectivo, de 6 meses de duración que finalizó el 20 de Noviembre de 2012. Se estudiaron todos los pacientes incidentes en la consulta de ERCA de nuestro hospital.

De la historia clínica digital de los pacientes se recogieron los datos demográ- ficos y epidemiológicos, así como las constantes vitales y los datos analíticos relacionados con el control de la PA. De igual forma se registraron los datos relacionados con la enfermedad renal crónica y su evolución así como la existencia de hábitos tóxicos para la salud. Estos datos se tomaron en la consulta inicial, a los tres y a los seis meses de permanencia en la consulta.

Para el estudio de las variables cualitativas usamos frecuencias absolutas y relativas. $Y$ para las cuantitativas utilizamos media y la desviación estándar o la mediana y el rango intercuartílico respectivamente. Para analizar la evolución temporal se usó modelos lineales generalizados para medidas repetidas (en el caso de normalidad) y Test de Freadman (no normales). Las comparaciones por pares se realizaron mediante la corrección de Bonfeorroni o la prueba de rangos con signos de Wilcoxon. Para la comparación inicial y final de las variables cualitativas dicotómicas utilizamos el test de McNemar.

El análisis de datos se realizó con el paquete estadístico IBM SPSS Statistics 19.0, estableciendo el nivel de significación en $p<0,05$.

\section{Resultados}

La muestra la formaron un total de 148 pacientes, de los cuales el $60,8 \%$ eran hombres $(n=90)$. La edad media fue $68,76 \pm 14,85$ años. El $21,6 \% \quad(n=32)$ tenían una Enfermedad Renal Crónica en estadio 3, el 74,3\% $(n=110)$ en estadio 4 , el $4,1 \%(n=6)$ en estadio 5 . El $45,3 \%(n=67)$ tenía diabetes mellitus. La Figura 1 muestra la etiología de la enfermedad renal crónica.

Evaluamos los cambios en las constantes vitales relacionadas con la presión arterial a lo largo del estudio, obteniendo diferencias significativas para la presión arte- 
rial sistólica ( $p<0,001 ;$ modelo lineal general), para la presión arterial diastólica $(p<0,001)$ y para la presión arterial media $(p<0,001)$, no siendo así para la frecuencia cardiaca $(p=0,307)$. (Tabla 1).

Tabla 1. Evolución de la presión arterial y la frecuencia cardiaca.

\begin{tabular}{|c|c|c|c|c|c|c|}
\hline & Inicial & pl & 3 meses & p2 & 6 meses & p3 \\
\hline $\begin{array}{l}\text { P.A. Sistólica } \\
(n=133)\end{array}$ & $\begin{array}{l}130,55 \\
+25,8\end{array}$ & $<0,001$ & $\begin{array}{r}121,10 \\
+38,08\end{array}$ & $<0,001$ & $\begin{array}{c}143 \\
+4,03\end{array}$ & $<0,001$ \\
\hline $\begin{array}{l}\text { P.A. Diastólica* } \\
(n=127)\end{array}$ & $\begin{array}{c}80 \\
{[67-88]}\end{array}$ & $<0,001$ & $\begin{array}{c}85 \\
{[75-125]}\end{array}$ & $<0,001$ & $\begin{array}{c}75 \\
{[65-83]}\end{array}$ & 0,001 \\
\hline $\begin{array}{l}\text { P.A. Media* } \\
(n=127)\end{array}$ & $\begin{array}{l}102,67 \\
{[80,67-} \\
119,67]\end{array}$ & $<0,001$ & $\begin{array}{c}77,67 \\
{[0-} \\
96,67]\end{array}$ & $<0,001$ & $\begin{array}{c}89,67 \\
{[75,33-} \\
107,67]\end{array}$ & $<0,001$ \\
\hline $\begin{array}{c}\text { Frecuencia } \\
\text { Cardiaca* } \\
(n=92)\end{array}$ & $\begin{array}{c}77,5 \\
{[66,25-} \\
86]\end{array}$ & - & $\begin{array}{c}74 \\
{[63-} \\
89,75]\end{array}$ & - & $\begin{array}{c}72 \\
{[62,25-} \\
85]\end{array}$ & - \\
\hline
\end{tabular}

P.A. Presión arterial. ${ }^{*} \mathrm{p}$ : Prueba de rangos con signos de Wilcoxon. $\mathrm{pl}$ : inicial vs 3 meses; p2: 3 meses vs 6 meses; p3: inicial vs 6 meses.

El número de hipotensores que utilizaron los pacientes se representa en la Figura 2. También existió una diferencia significativa en el descenso de peso de los pacientes $(n=127)$ que pasaron de un peso de $80,13 \pm 16,76 \mathrm{~kg}$ a $77,64 \pm 16,18 \mathrm{~kg}$ al final del seguimiento $(p<0,001)$. La presencia de edemas $(n=138)$ varió de un $26,1 \%$ a un $18,8 \%$ al concluir el estudio $(p=0,052)$

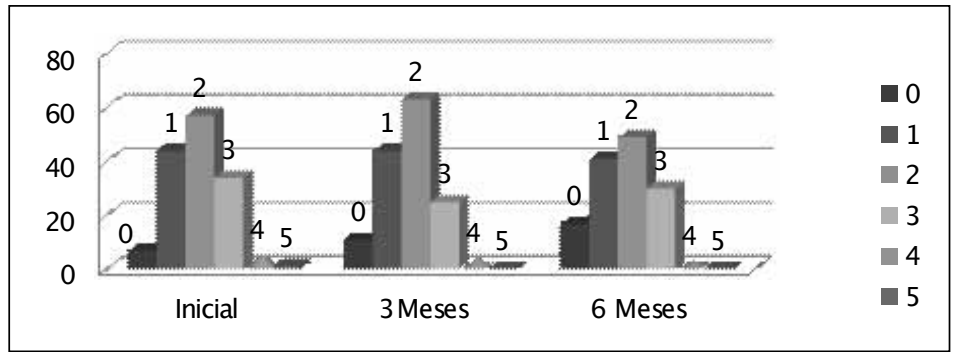

Figura 2. Número de hipotensos.

Analizamos el sodio en orina $(p=0,665)$, como marcador de la ingesta de sodio en la dieta y observamos que no hubo diferencias significativas.

Encontramos cambios significativos en el tabaco $(p=0,004)$ no siendo así con el consumo de alcohol $(p=0,5)$ (Tabla 2).

Tabla 2. Hábitos tóxicos.

\begin{tabular}{|c|c|c|c|}
\hline & Inicial & 6 meses & $p$ \\
\hline Tabaco & $16,5 \%(n=23)$ & $9,8 \%(n=14)$ & 0,004 \\
\hline Alcohol & $11 \%(n=16)$ & $9,7 \%(n=14)$ & 0,5 \\
\hline
\end{tabular}

1p: Prueba de McNemar.

\section{Discusión}

Existen muchos factores de riesgo que repercuten en la progresión de la enfermedad renal como puede ser la proteinuria elevada, la dislipemia, hábitos tóxicos y en el caso de los diabéticos, un mal control de la misma. Todos estos factores conforman un complejo mecanismo de causas añadida a la enfermedad renal crónica que si no son controladas adecuadamente, hacen que las pocas nefronas que aún funcionan en este estadio de la enfermedad renal se vayan perdiendo con más rapidez ${ }^{1}$.

Por otro lado, la prevalencia de la hipertensión arterial en los pacientes con enfermedad renal crónica, aumenta a medida que desciende el filtrado glomerular². Una elevada presión arterial produce arteriosclerosis de las arterias intertubulares y arteriolas aferentes, disminuyendo el flujo renal ${ }^{5}$.

Actualmente, nadie pone en duda que la enfermería nefrológica debe estar presente en las consultas de ERCA, ya que desarrolla un trabajo fundamental, estando directamente implicada en el control de casi todos los factores anteriormente mencionados y muy directamente sobre el control de la presión arterial, motivo de este trabajo.

En nuestra población, hemos de sumar a la hipertensión otro factor de riesgo como es la edad de la población estudiada, que por sí misma supone otro factor de riesgo para la enfermedad renal crónica, más aún si tenemos en cuenta la elevada prevalencia de pacientes diabéticos. Por lo que las intervenciones de enfermería no se pueden limitar a las recogidas en este trabajo, si no que se ampliaron para poder corregir todos aquellos factores de riesgo que presentaban nuestros pacientes.

Las actuaciones de enfermería que se han realizado en la consulta, han favorecido un mejor control de la presión arterial sistólica, diastólica y media, aunque no se consiguió disminuir significativamente el número de hipotensores.

Estas actuaciones resultaron también efectivas al conseguir disminuir el peso medio de los pacientes, los edemas y el consumo de tabaco.

El sodio en orina se utilizó como marcador de la ingesta de sodio de los pacientes, y a pesar de ser una constante 
su restricción en el tratamiento de los pacientes hipertensos no hemos logrado disminuir su consumo de forma significativa.

Esto podría deberse a que muchos de los pacientes que recibimos en la consulta ERCA, y que inician una dieta hipoproteica y al mismo tiempo afrontan en un futuro próximo un tratamiento renal sustitutivo, pasan por una fase depresiva que cursa con anorexia. Desde la consulta flexibilizamos un poco la restricción de sodio con el objetivo que el paciente al menos haga la dieta prescrita más apetecible reduciéndolo progresivamente. El consumo de alcohol tampoco ha mejorado sensiblemente.

Observamos que los resultados mejoran significativamente a la entrada en la consulta y a los tres meses, y aunque a los seis meses la mejoría sigue siendo significativas, la diferencia es menor que la inicial, lo que nos hace pensar que enfermería no debe de bajar la guardia y reforzar la información que proporcionamos siempre que sea necesario.

Podemos concluir que las actividades de enfermería que se desarrollan en la consulta ERCA han mejorado el control de la presión arterial, obteniendo el efecto deseado. Hemos observado que debemos mantener los consejos sanitarios a lo largo del tiempo para mantener sus efectos, ya que algunos factores de riesgo como el consumo de sal o de alcohol no han demostrado los resultados esperado, y que con el tiempo, si no se refuerzan pueden perder eficacia, por lo que las intervenciones de enfermería deben ser una constante en el tratamiento del paciente hipertenso con ERCA.
Recibido: 4 Febrero 2014

Revisado: 6 Febrero 2014

Modificado: 6 Febrero 2014

Aceptado: 10 Febrero 2014

\section{Bibliografía}

1. Arteaga, J.M. Hipertensión arterial e insuficiencia renal. ANALEZ Sis. San. Navarra. 1998. Vol. 21, Suplemento 1.

2. http://www. Guías de actuación de enfermería en hipertensión arterial y riesgos cardiovasculares asociados. EHRICA. Marzo 2013.

3. Luis Rodrigo, M.T. Los diagnósticos enfermeros. $7^{\mathrm{a}}$ edición (2006). Barcelona: MASSON.

4. Alonso Nates, R. Pelayo Alonso, R. Manual de enfermería nefrológica. Pulso ediciones. Barcelona. 2012.

5. Sociedad Española de Hipertensión/Liga Española para la lucha contra la Hipertensión Arterial SHELEHLA. Guías Española de Hipertensión Arterial 2005. Hipertensión 2005; Supl 2 47-57.

6. Guías SEN "Riñón y Enfermedad cardiovascular". Nefrología 2004: Vol 24. Supl 6. 